\title{
Cobalt-catalyzed peri-selective alkoxylation of 1-naphthylamine derivatives
}

\author{
Jiao-Na Han, Cong Du, Xinju Zhu, Zheng-Long Wang, Yue Zhu, Zhao-Yang Chu, \\ Jun-Long Niu* and Mao-Ping Song*
}

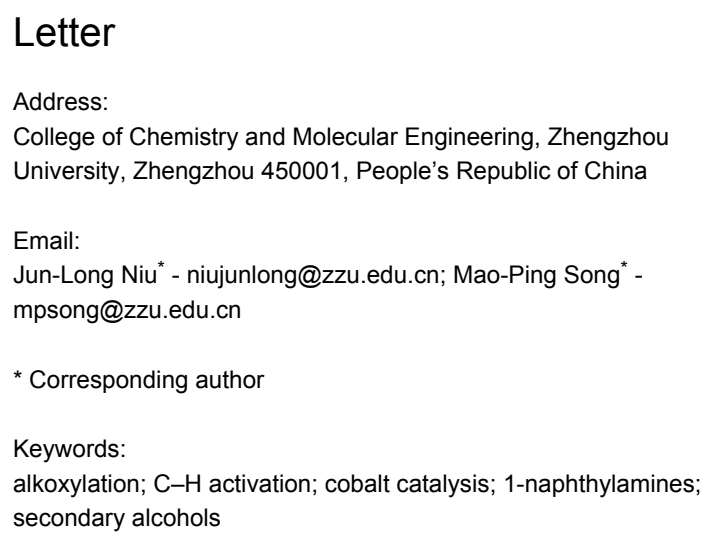

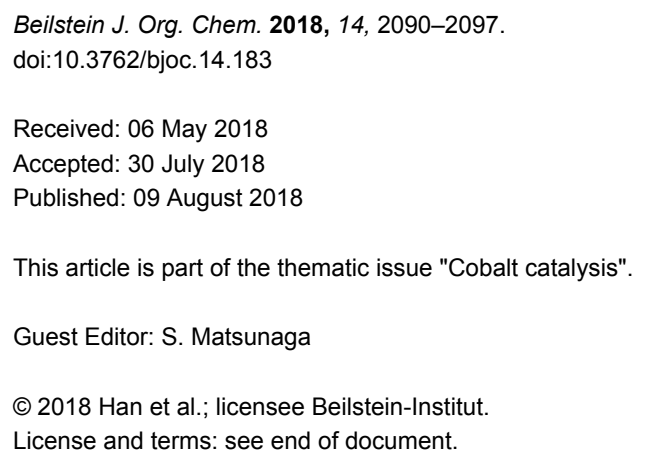

\begin{abstract}
A cobalt-catalyzed $\mathrm{C}\left(\mathrm{sp}^{2}\right)-\mathrm{H}$ alkoxylation of 1-naphthylamine derivatives has been disclosed, which represents an efficient approach to synthesize aryl ethers with broad functional group tolerance. It is noteworthy that secondary alcohols, such as hexafluoroisopropanol, isopropanol, isobutanol, and isopentanol, were well tolerated under the current catalytic system. Moreover, a series of biologically relevant fluorine-aryl ethers were easily obtained under mild reaction conditions after the removal of the directing group.
\end{abstract}

\section{Introduction}

Aryl ethers are common structural units present in many natural products, functional materials, and pharmaceuticals [1]. Consequently, a variety of strategies have emerged to access them, including $\mathrm{Pd}$-catalyzed and $\mathrm{Cu}$-catalyzed coupling reactions (Buchwald-Harting couplings and Ullmann reactions) [2-4]. However, these classic methods always possess some limitations such as preactivated starting materials, poor regioselectivities, and tedious steps [5]. Therefore, it is desirable to develop an effective strategy to achieve this transformation $[6,7]$.

Over the past few decades, transition-metal-catalyzed $\mathrm{C}-\mathrm{H}$ activation to form $\mathrm{C}-\mathrm{C}$ or $\mathrm{C}$-heteroatom bonds has attracted more attention [8-13]. In particular, the formation of $\mathrm{C}-\mathrm{O}$ bonds is widely used in the syntheses of pharmaceuticals and functional materials [14-17]. The direct hydroxylation [18,19] and acetoxylation [20-22] have been developed rapidly in recent years. By contrast, alkoxylation or phenoxylation confronts great challenges because alkanols or phenols are easily converted into the corresponding aldehydes, ketones, or carboxylic acids [7,23-25]. Recently, Gooßen [26,27], Sanford [28], Song, $[29,30]$ and others [31-42] have successfully reported alkoxylation reactions with the auxiliary of directing groups. However, the transition-metal-catalyzed $\mathrm{C}-\mathrm{H}$ alkoxylation is still largely limited to palladium- [28,33-40] or copper- [26,27,29,41,42] catalyzed systems. Recently, the inexpensive cobalt catalysts have received significant attention because of their unique and 
versatile activities in the $\mathrm{C}-\mathrm{H}$ functionalizations [43-51]. In 2015 , the cobalt-catalyzed alkoxylation of aromatic (and olefinic) carboxamides with primary alcohols was first reported by the Niu and Song group (Figure 1a) [30]. Successively, Ackermann realized the electrochemical cobalt-catalyzed alkoxylation via a similar process (Figure 1b) [32]. However, cobalt-catalyzed directed coupling of arenes with secondary alcohols has not been reported so far. Herein, we explored a simple and facile protocol for cobalt-catalyzed picolinamidedirected alkoxylation of 1-naphthylamine derivatives with alcohols (Figure 1c).

\section{Results and Discussion}

Initially, $N$-(naphthalen-1-yl)picolinamide (1a) and hexafluoroisopropanol (HFIP, 2a) were chosen as the model substrates to optimize the alkoxylation reaction (Table 1, see more in Tables S1-S5 in Supporting Information File 1). To our delight, the desired product $3 \mathbf{a a}$ was obtained in $66 \%$ yield when using $\mathrm{Co}(\mathrm{OAc})_{2} \cdot 4 \mathrm{H}_{2} \mathrm{O}$ as catalyst and $\mathrm{Ag}_{2} \mathrm{CO}_{3}$ as oxidant (Table 1, entry 1). Other cobalt salts such as $\mathrm{CoF}_{3}$ and $\mathrm{CoF}_{2}$ were also employed as metal catalysts for C8 alkoxylation of $\mathbf{1 a}$, and $\mathrm{CoF}_{2}$ was proved to be the optimal catalyst, affording 3aa in $71 \%$ yield (Table 1, entries 2 and 3). Subsequently, various bases such as $\mathrm{Na}_{2} \mathrm{CO}_{3}, \mathrm{~K}_{2} \mathrm{CO}_{3}$, and $\mathrm{Cs}_{2} \mathrm{CO}_{3}$ were screened (Table 1, entries 4-6), which indicated that $\mathrm{Cs}_{2} \mathrm{CO}_{3}$ was most effective and the alkoxylated product $\mathbf{3}$ aa could be isolated in
$82 \%$ yield. Next, the effect of oxidants on the reactivity was investigated, and $\mathrm{Ag}_{2} \mathrm{CO}_{3}$ showed a superior result compared with alternative oxidants (Table 1, entries 6-8). Moreover, DCE and HFIP as co-solvents demonstrated higher reactivity, resulting in a slightly increased yield in 84\% (Table 1, entries 9-11). Finally, variation of the reaction temperature did not promote the reaction efficiency.

With the established alkoxylated protocol in hand, the substrate scope of 1-naphthylamine derivatives was explored as shown in Scheme 1. Halogenated naphthylamines could afford the target products in $86-88 \%$ yields (3ba-3ca). Nitro- (1d) and benzenesulfonyl- (1e) substituted naphthylamines were found to proceed smoothly via this strategy (61-64\%). In addition, a disubstituted naphthylamine provided the alkoxylated product in $47 \%$ yield (3fa). Moreover, a Boc amino group at C5 of the substrate $\mathbf{1 g}$ was also compatible with the transformation (33\%). When a methoxy group was located at the C7 site of the naphthylamine, sterically hindered product 3 ha was obtained in $81 \%$. Besides, some benzylamine derivatives ( $N$ - $(1-$ phenylethyl)picolinamide and $N$-benzhydrylpicolinamide) were attempted. However, no desired product could be detected.

Next, the substrate scope of alcohols was investigated. As shown in Scheme 2, both primary and secondary alcohols were compatible with the slightly modified optimized conditions. A

(a)<smiles>[R]Oc1ccccc1C(=O)Nc1cccc[n+]1[O-]</smiles>

(b)<smiles>O=C(Nc1cccc[n+]1[O-])c1ccccc1</smiles>
$+\mathrm{ROH}$

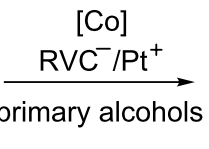<smiles>[R]Oc1ccccc1C(=O)Nc1cccc[n+]1[O-]</smiles>

(c)<smiles>[R1]c1cccc2ccccc12</smiles><smiles>[R]</smiles><smiles>[R]C([R])O</smiles>
[Co]<smiles></smiles>

- inexpensive cobalt catalyst

- good functional group tolerance

- primary and secondary alcohols

- C8 selective alkoxylation 
Table 1: Optimization of the reaction conditions. ${ }^{a}$

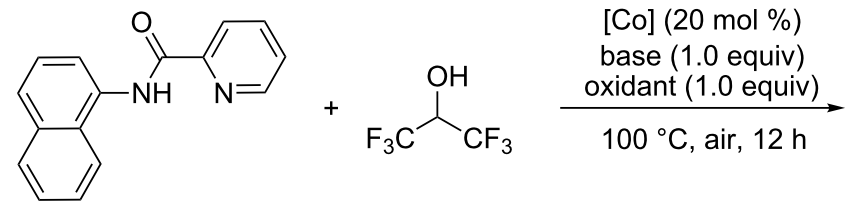

1 a

$2 a$

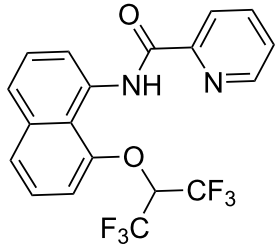

3aa

\begin{tabular}{ccll}
\hline entry & catalyst & base & oxidant \\
\hline 1 & $\mathrm{Co}(\mathrm{OAc})_{2} \cdot 4 \mathrm{H}_{2} \mathrm{O}$ & $t$-AmONa & $\mathrm{Ag}_{2} \mathrm{CO}_{3}$ \\
2 & $\mathrm{CoF}_{3}$ & $t$-AmONa & $\mathrm{Ag}_{2} \mathrm{CO}_{3}$ \\
3 & $\mathrm{CoF}_{2}$ & $t$-AmONa & $\mathrm{Ag}_{2} \mathrm{CO}_{3}$ \\
4 & $\mathrm{CoF}_{2}$ & $\mathrm{Na}_{2} \mathrm{CO}_{3}$ & $\mathrm{Ag}_{2} \mathrm{CO}_{3}$ \\
5 & $\mathrm{CoF}_{2}$ & $\mathrm{~K}_{2} \mathrm{CO}_{3}$ & $\mathrm{Ag}_{2} \mathrm{CO}_{3}$ \\
6 & $\mathrm{CoF}_{2}$ & $\mathrm{Cs}_{2} \mathrm{CO}_{3}$ & $\mathrm{Ag}_{2} \mathrm{CO}_{3}$ \\
7 & $\mathrm{CoF}_{2}$ & $\mathrm{Cs}_{2} \mathrm{CO}_{3}$ & $\mathrm{AgNO}_{3}$ \\
8 & $\mathrm{CoF}_{2}$ & $\mathrm{Cs}_{2} \mathrm{CO}_{3}$ & $\mathrm{Ag}_{2} \mathrm{O}$ \\
$9^{\mathrm{b}}$ & $\mathrm{CoF}_{2}$ & $\mathrm{Cs}_{2} \mathrm{CO}_{3}$ & $\mathrm{Ag}_{2} \mathrm{CO}_{3}$ \\
$10^{\mathrm{c}}$ & $\mathrm{CoF}_{2}$ & $\mathrm{Cs}_{2} \mathrm{CO}_{3}$ & $\mathrm{Ag}_{2} \mathrm{CO}_{3}$ \\
$11^{\mathrm{d}}$ & $\mathrm{CoF}_{2}$ & $\mathrm{Cs}_{2} \mathrm{CO}_{3}$ & $\mathrm{Ag}_{2} \mathrm{CO}_{3}$ \\
\hline
\end{tabular}

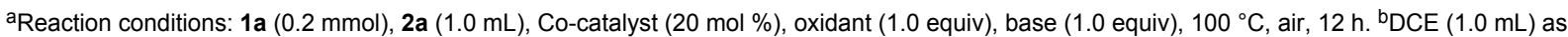
co-solvent. ${ }^{\mathrm{PhCF}} 3(1.0 \mathrm{~mL})$ as co-solvent. ${ }^{\mathrm{d}} \mathrm{PhF}(1.0 \mathrm{~mL})$ as co-solvent. $\mathrm{DCE}=1,2$-dichloroethane.

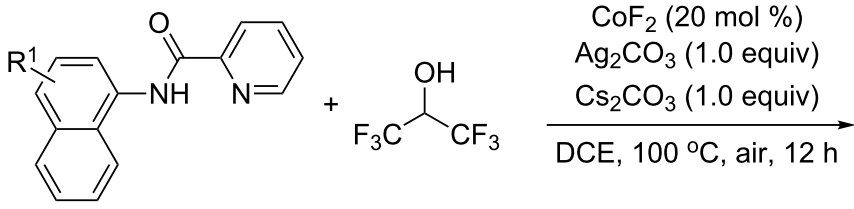

$1 \mathrm{a}-\mathrm{h}$

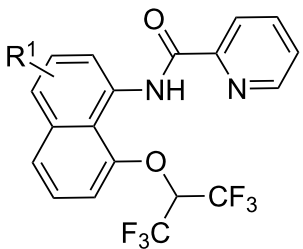

3

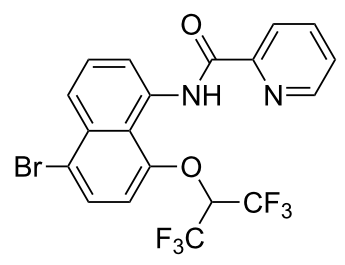

3ba, $88 \%$<smiles>O=C(Nc1ccc(Br)c2cccc(OC(F)(F)C(F)(F)F)c12)c1ccccn1</smiles>

3ca, $86 \%$<smiles>O=C(Nc1ccc([N+](=O)[O-])c2cccc(OC(F)(F)C(F)(F)F)c12)c1ccccn1</smiles>

3da, $64 \%$

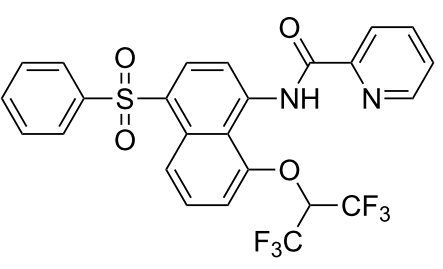

3ea, $61 \%$

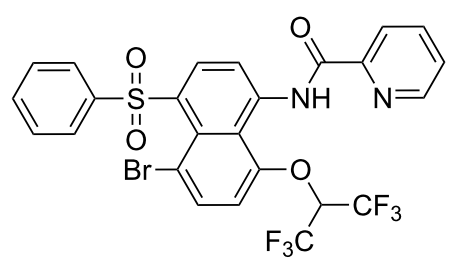

3fa, $47 \%$

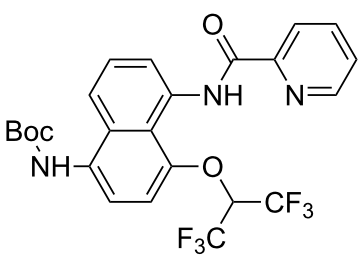

3ga, $33 \%$

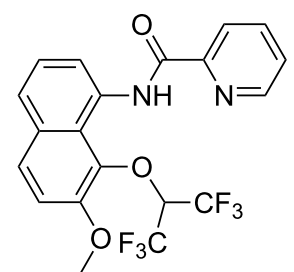

3ha, $81 \%$

Scheme 1: Reaction scope with respect to $\mathrm{N}$-(naphthalen-1-yl)picolinamide derivatives. Reaction conditions: 1 (0.2 mmol), $2 \mathrm{a}(1.0 \mathrm{~mL}), \mathrm{CoF}_{2}$ (20 mol \%), $\mathrm{Ag}_{2} \mathrm{CO}_{3}$ (1.0 equiv), $\mathrm{Cs}_{2} \mathrm{CO}_{3}$ (1.0 equiv), DCE $(1.0 \mathrm{~mL}), 100^{\circ} \mathrm{C}$, air, $12 \mathrm{~h}$. 


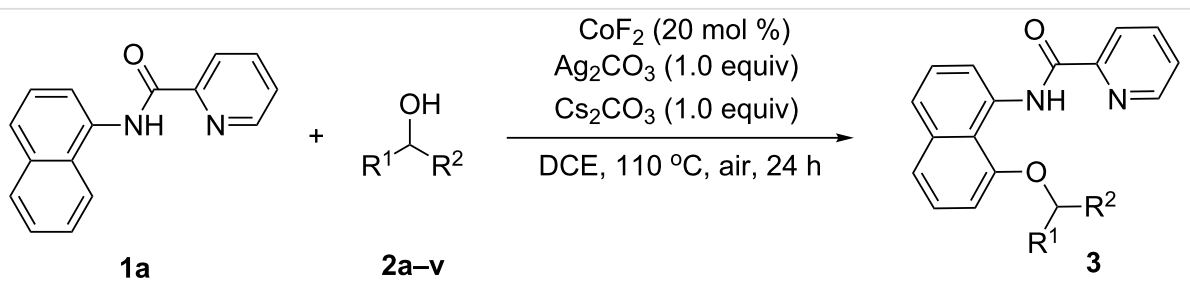

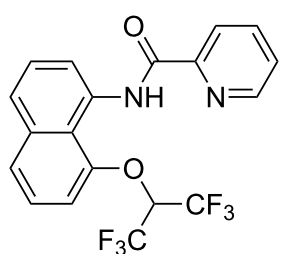

3aa, $84 \%$

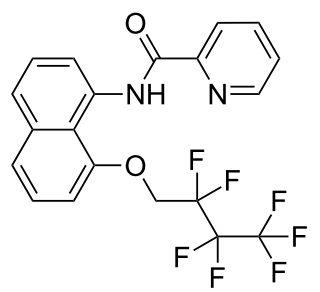

3ae, $73 \%$

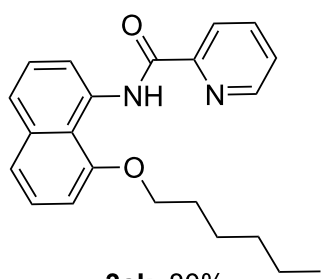

3ak, $80 \%$

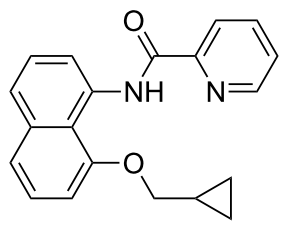

$3 a o, 45 \%$

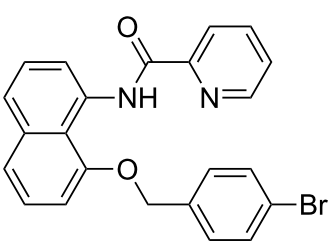

3as, $70 \%$

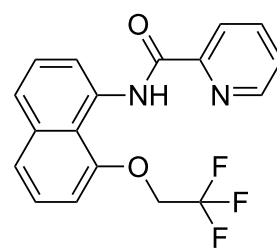

$3 a b, 76 \%$

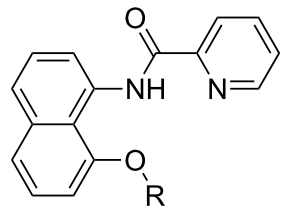

$\mathrm{R}=$ Me, 3af, $54 \%$

$\mathrm{R}=\mathrm{Et}, \mathbf{3 a g}, 80 \%$

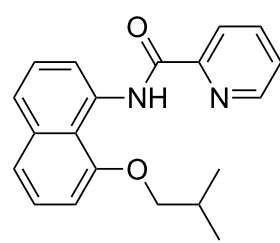

3al, $89 \%$

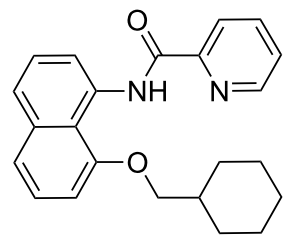

3ap, $46 \%$

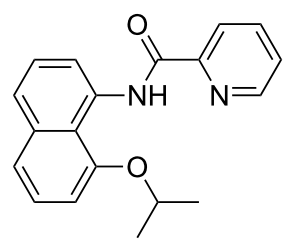

3at, $58 \%$

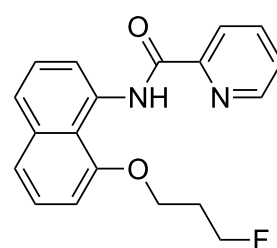

3ac, $77 \%$

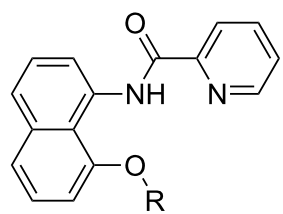

$\mathrm{R}=\mathrm{Pr}, \mathbf{3 a h}, 66 \%$

$\mathrm{R}=\mathrm{Bu}, 3 \mathbf{a i}, 84 \%$

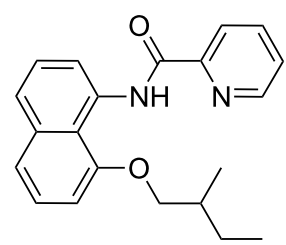

3am, 66\%

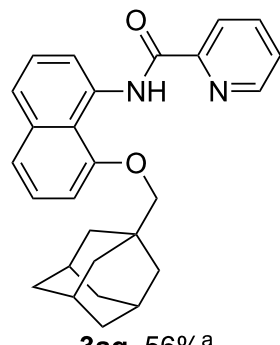

3aq, 56\%a

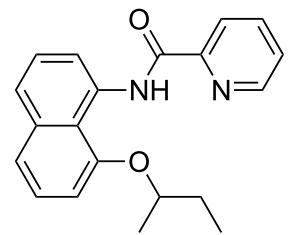

3au, 65\%
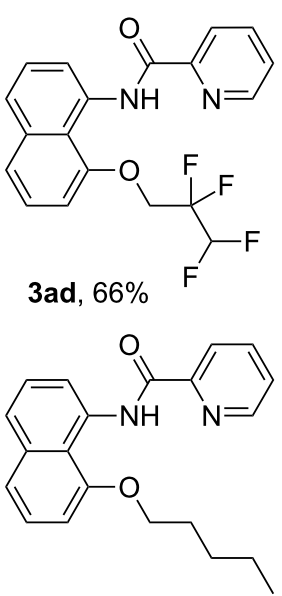

3aj, 78\%

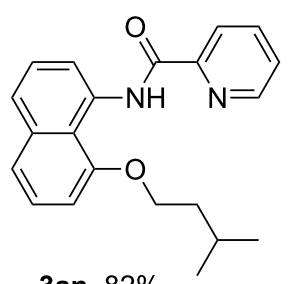

3an, $82 \%$

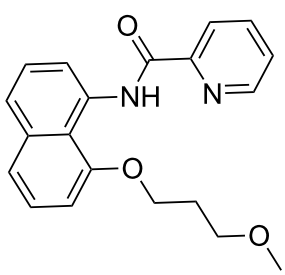

3ar, $68 \%^{\mathrm{b}}$

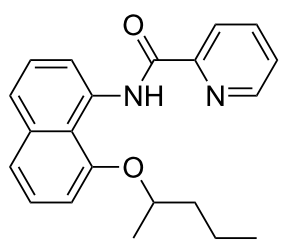

3av, 89\%

Scheme 2: Reaction scope with respect to alcohols. Reaction conditions: 1 a $(0.2 \mathrm{mmol}), 2(1.0 \mathrm{~mL}), \mathrm{CoF}_{2}\left(20 \mathrm{~mol}^{\circ}\right), \mathrm{Ag}_{2} \mathrm{CO}_{3}(1.0 \mathrm{equiv}), \mathrm{Cs}_{2} \mathrm{CO}_{3}$ (1.0 equiv), DCE $(1.0 \mathrm{~mL}), 110{ }^{\circ} \mathrm{C}$, air, $24 \mathrm{~h} .{ }^{\mathrm{a}} 2 \mathrm{q}$ or $2 \mathrm{~s}\left(5.0\right.$ equiv). ${ }^{\mathrm{b}} 48 \mathrm{~h}$.

variety of fluoro-substituted alcohols $\mathbf{2} \mathbf{a}-\mathbf{e}$ proceeded smoothly to afford the corresponding products in moderate to good yields (66-84\%). Simple primary alkyl alcohols were well tolerated to provide the desired products in $54-84 \%$ yields (3af-ak). Also, the branched alcohols $\mathbf{2} \mathbf{l}, \mathbf{2} \mathbf{m}$, and $\mathbf{2 n}$ were employed to afford the corresponding products in 66-89\% yields. Cyclopropyl- 
methanol (2o), cyclohexylmethanol (2p), and adamantanemethanol (2q) were compatible with the transformation (45-56\%). Moreover, an aliphatic ether and benzyl alcohol were proved to be effective coupling partners to provide 3ar and 3 as in $68 \%$ and $70 \%$ yields, respectively. Compared with Co-catalyzed alkoxylation of arenes with primary alcohols [30,31], HFIP (2a), isopropanol (2t), isobutanol (2u), and isopentanol $(2 \mathbf{v})$ could all proceed smoothly to deliver the alkoxylated products in $58-89 \%$ yields. Furthermore, we attempted some tertiary alcohols (tert-butanol and 2-methyl-2butanol). However, no desired product could be detected.

In order to study the reaction mechanism, a series of control experiments were carried out (Scheme 3, see more details in Supporting Information File 1). In the absence of cobalt salt, no product was obtained under the standard reaction conditions. Under an argon atmosphere and without $\mathrm{Ag}_{2} \mathrm{CO}_{3}$, the product was isolated in $12 \%$ yield when a stoichiometric amount of $\mathrm{CoF}_{3}$ was introduced, whereas no product was obtained in the presence of $\mathrm{CoF}_{2}$ (Scheme 3, reaction 1). These results imply that the reaction should initiates from a $\mathrm{Co}^{\mathrm{III}}$ species. The addition of a radical quencher, benzoquinone (BQ), suppressed the formation of product 3aa. When 2,2,6,6-tetramethyl-1piperidinyloxyl (TEMPO) or 2,6-di-tert-butyl-4-methylphenol (BHT) was added under the standard reaction conditions, a significantly reduced yield (39\% or $23 \%$ ) was obtained (Scheme 3 , reaction 2). These facts suggest that a radical approach may be involved in the reaction. Moreover, in the parallel experiments, a KIE value of 1.1 was observed between $\mathbf{1 a}$ or $\left[\mathrm{D}_{1}\right]-\mathbf{1 a}$ with 2a, which indicates that Co-catalyzed $\mathrm{C}-\mathrm{H}$ bond cleavage should not be the rate determining step (Scheme 3, reaction 3).

On the basis of the above studies and previous literature $[30,31,46,47]$, a plausible reaction mechanism for cobalt-catalyzed alkoxylation was proposed. As shown in Scheme 4, initially, $\mathrm{Co}^{\mathrm{II}} \mathrm{X}_{2}$ could be oxidized to $\mathrm{Co}^{\mathrm{III}} \mathrm{X}_{2} \mathrm{OR}$ in the presence of a silver salt and an alcohol. Based on the experiments and the density functional theory calculations (DFT) [30,31],<smiles>O=C(Nc1cccc2ccccc12)c1ccccn1</smiles>

1a<smiles>OC(C(F)(F)F)C(F)(F)F</smiles>

2a

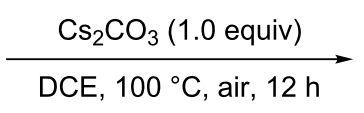

DCE, $100^{\circ} \mathrm{C}$, air, $12 \mathrm{~h}$<smiles>O=C(Nc1cccc2cccc(OC(C(F)(F)F)C(F)(F)F)c12)c1ccccn1</smiles>

3aa

w/o [Co] $\mathrm{Ag}_{2} \mathrm{CO}_{3}$ (1.0 equiv), air n.r. w/o $[\mathrm{Ag}] \quad \mathrm{CoF}_{3}$ (1.0 equiv), $\mathrm{Ar} \quad 12 \%$ $\mathrm{CoF}_{2}(20 \mathrm{~mol} \%), \mathrm{Ar} \quad$ n.r.<smiles>O=C(Nc1cccc2ccccc12)c1ccccn1</smiles>

$1 \mathbf{a}$<smiles>O=C(Nc1cccc2ccccc12)c1ccccn1</smiles>

$1 a$<smiles>[2H]c1cccc2cccc(NC(=O)c3ccccn3)c12</smiles>

$\left[D_{1}\right]-1 \mathbf{a}$

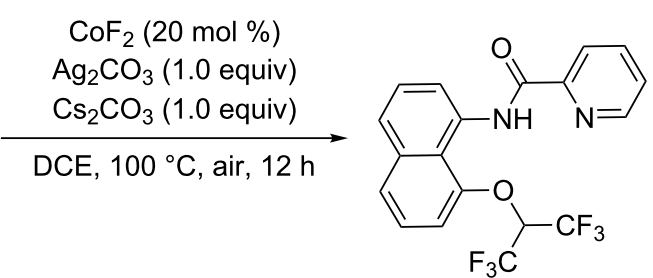

3aa $\begin{array}{lr}\text { BQ }(1.0 \text { equiv) } & \text { n.r. } \\ \text { TEMPO (1.0 equiv) } & 39 \% \\ \text { BHT (1.0 equiv) } & 23 \%\end{array}$ 


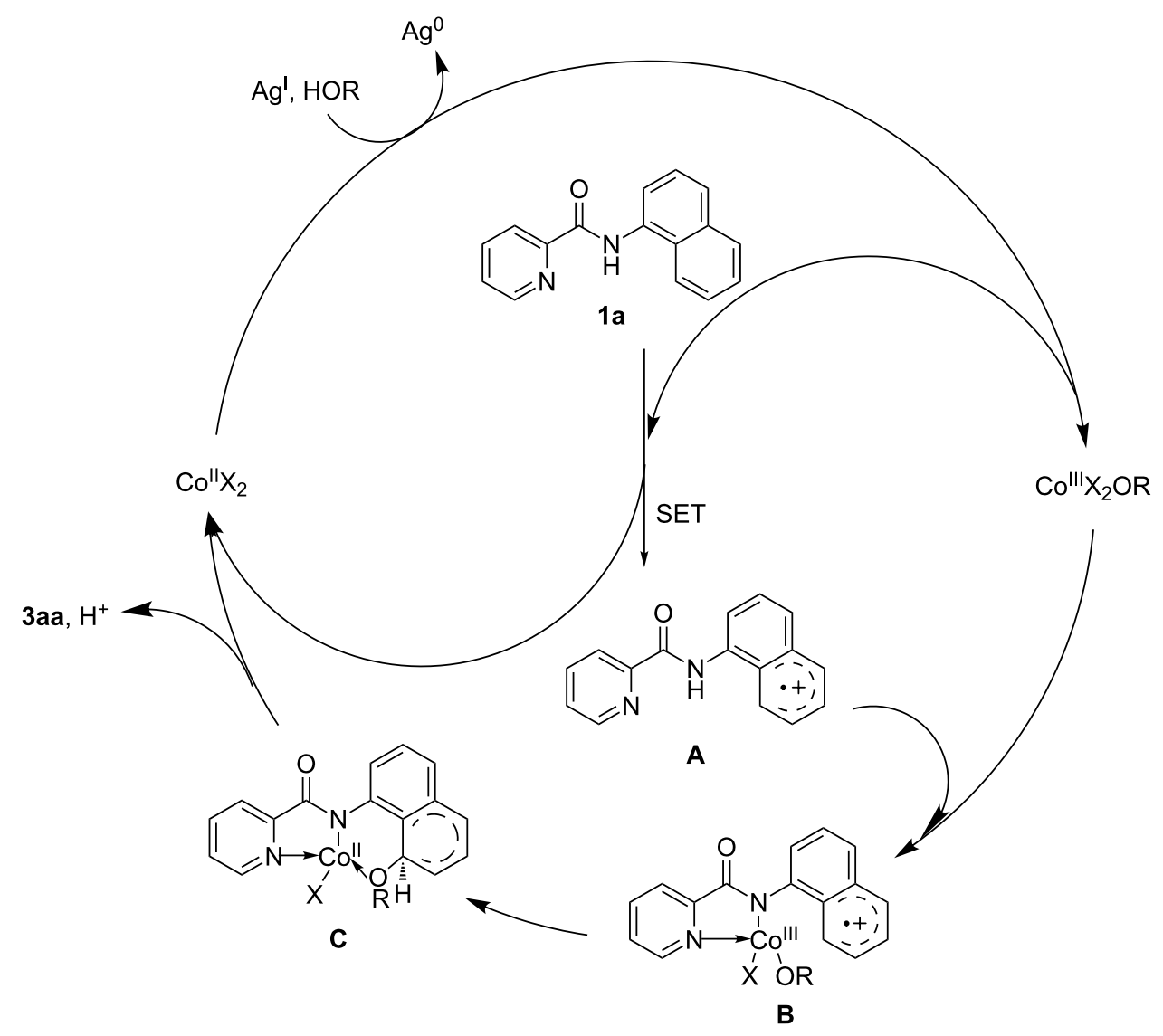

Scheme 4: Proposed reaction mechanism

the $\mathrm{C}-\mathrm{H}$ activation most possibly proceeded via a single-electron transfer (SET) path compared to a concerted metalation-deprotonation (CMD) path. Followed by an intermolecular SET process, the cation-radical intermediate $\mathbf{A}$ was generated, which coordinates with a $\mathrm{Co}^{\mathrm{III}}$ species to provide the intermediate $\mathbf{B}$. Subsequently, the transfer of ligand OR from the coordinated $\mathrm{Co}^{\mathrm{III}}$ to the naphthalene ring led to the formation of intermediate $\mathbf{C}$. Finally, the alkoxylated product 3aa was released accompanied with the deprotonation and the $\mathrm{Co}^{\mathrm{II}}$ species was transformed into the $\mathrm{Co}^{\mathrm{III}}$ species by re-oxidization.

The current alkoxylation methodology also exhibited potential applications. When treated with $\mathrm{NaOH}$ at $80^{\circ} \mathrm{C}$, the picolinic acid directing group could be easily removed, and the corresponding 4 was obtained in $88 \%$ yield (Scheme 5).

\section{Conclusion}

In summary, a cobalt-catalyzed C8 alkoxylation of naphthylamine derivatives with both primary and secondary alcohols was developed. This protocol is characterized by mild reaction conditions, broad substrate scope, and good functional group

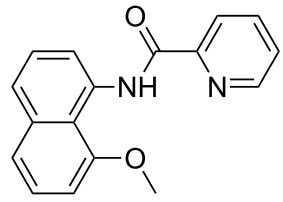

3af

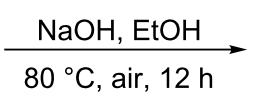

$80^{\circ} \mathrm{C}$, air, $12 \mathrm{~h}$

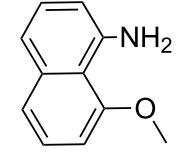

$4,88 \%$
Scheme 5: Removal of the directing group.

tolerance. Moreover, the excellent compatibility of fluorinesubstituted alcohols (for instance, HFIP, trifluoroethanol, and 3-fluoropropanol etc.) shows that this strategy is highly valuable for the syntheses of biologically relevant fluorine-aryl ethers after the removal of the directing group. The above studies of the mechanism indicate that this reaction undergoes a SET process and cobalt salt is the actual catalyst. Overall, this protocol provides a new insight into the cobalt-catalyzed alkoxylation of naphthylamine derivatives. Further exploration of this strategy to aliphatic substrates is currently in progress. 


\section{Supporting Information}

\section{Supporting Information File 1}

Experimental details and characterization data of new compounds, and X-ray crystal structure details for 3aa. [https://www.beilstein-journals.org/bjoc/content/ supplementary/1860-5397-14-183-S1.pdf]

\section{Supporting Information File 2}

Crystallographic information file for compound 3aa. [https://www.beilstein-journals.org/bjoc/content/ supplementary/1860-5397-14-183-S2.cif]

\section{Acknowledgements}

The work was supported by the National Natural Science Foundation of China (Nos. 21772179, 21502173, 21672192), Outstanding Young Talent Research Fund of Zhengzhou University (No. 1521316002).

\section{$\mathrm{ORCID}^{\circledR} \mathrm{iDs}$}

Jun-Long Niu - https://orcid.org/0000-0001-8012-4676 Mao-Ping Song - https://orcid.org/0000-0003-3883-2622

\section{References}

1. Enthaler, S.; Company, A. Chem. Soc. Rev. 2011, 40, 4912-4924. doi:10.1039/c1cs15085e

2. Altman, R. A.; Shafir, A.; Choi, A.; Lichtor, P. A.; Buchwald, S. L. J. Org. Chem. 2008, 73, 284-286. doi:10.1021/jo702024p

3. Hartwig, J. F. Nature 2008, 455, 314-322. doi:10.1038/nature07369

4. Monnier, F.; Taillefer, M. Angew. Chem., Int. Ed. 2009, 48, 6954-6971. doi:10.1002/anie.200804497

5. Bruice, T. C. J. Am. Chem. Soc. 1957, 79, 702-705. doi:10.1021/ja01560a055

6. Beccalli, E. M.; Broggini, G.; Martinelli, M.; Sottocornola, S. Chem. Rev. 2007, 107, 5318-5365. doi:10.1021/cr068006f

7. Chen, X.; Hao, X.-S.; Goodhue, C. E.; Yu, J.-Q. J. Am. Chem. Soc. 2006, 128, 6790-6791. doi:10.1021/ja061715q

8. Colby, D. A.; Bergman, R. G.; Ellman, J. A. Chem. Rev. 2010, 110, 624-655. doi:10.1021/cr900005n

9. Xiao, B.; Gong, T.-J.; Liu, Z.-J.; Liu, J.-H.; Luo, D.-F.; Xu, J.; Liu, L. J. Am. Chem. Soc. 2011, 133, 9250-9253. doi:10.1021/ja203335u

10. Wei, Y.; Yoshikai, N. Org. Lett. 2011, 13, 5504-5507. doi:10.1021/ol202229w

11. John, A.; Nicholas, K. M. J. Org. Chem. 2011, 76, 4158-4162. doi:10.1021/jo200409h

12. Zhao, J.; Wang, Y.; He, Y.; Liu, L.; Zhu, Q. Org. Lett. 2012, 14, 1078-1081. doi:10.1021/ol203442a

13. Zhang, C.; Feng, P.; Jiao, N. J. Am. Chem. Soc. 2013, 135 , 15257-15262. doi:10.1021/ja4085463

14. Huang, R.; Huang, Y.; Lin, X.; Rong, M.; Weng, Z. Angew. Chem., Int. Ed. 2015, 54, 5736-5739. doi:10.1002/anie.201501257

15. Shan, G.; Yang, X.; Zong, Y.; Rao, Y. Angew. Chem., Int. Ed. 2013, 52, 13606-13610. doi:10.1002/anie.201307090
16. Yan, Y.; Feng, P.; Zheng, Q.-Z.; Liang, Y.-F.; Lu, J.-F.; Cui, Y.; Jiao, N. Angew. Chem., Int. Ed. 2013, 52, 5827-5831. doi:10.1002/anie.201300957

17. Krylov, I. B.; Vil', V. A.; Terent'ev, A. O. Beilstein J. Org. Chem. 2015, 11, 92-146. doi:10.3762/bjoc.11.13

18. Zhang, Y.-H.; Yu, J.-Q. J. Am. Chem. Soc. 2009, 131, 14654-14655. doi:10.1021/ja907198n

19. Li, X.; Liu, Y.-H.; Gu, W.-J.; Li, B.; Chen, F.-J.; Shi, B.-F. Org. Lett. 2014, 16, 3904-3907. doi:10.1021/ol5016064

20. Dick, A. R.; Hull, K. L.; Sanford, M. S. J. Am. Chem. Soc. 2004, 126, 2300-2301. doi:10.1021/ja031543m

21. Cheng, X.-F.; Li, Y.; Su, Y.-M.; Yin, F.; Wang, J.-Y.; Sheng, J.; Vora, H. U.; Wang, X.-S.; Yu, J.-Q. J. Am. Chem. Soc. 2013, 135, 1236-1239. doi:10.1021/ja311259x

22. Emmert, M. H.; Cook, A. K.; Xie, Y. J.; Sanford, M. S. Angew. Chem., Int. Ed. 2011, 50, 9409-9412. doi:10.1002/anie.201103327

23. Hoover, J. M.; Ryland, B. L.; Stahl, S. S. J. Am. Chem. Soc. 2013, 135, 2357-2367. doi:10.1021/ja3117203

24. Kumpulainen, E. T. T.; Koskinen, A. M. P. Chem. - Eur. J. 2009, 15, 10901-10911. doi:10.1002/chem.200901245

25. Okada, T.; Asawa, T.; Sugiyama, Y.; Kirihara, M.; Iwai, T.; Kimura, Y. Synlett 2014, 25, 596-598. doi:10.1055/s-0033-1340483

26. Bhadra, S.; Dzik, W. I.; Gooßen, L. J. Angew. Chem., Int. Ed. 2013, 52, 2959-2962. doi:10.1002/anie.201208755

27. Bhadra, S.; Matheis, C.; Katayev, D.; Gooßen, L. J. Angew. Chem., Int. Ed. 2013, 52, 9279-9283. doi:10.1002/anie.201303702

28. Desai, L. V.; Malik, H. A.; Sanford, M. S. Org. Lett. 2006, 8 , 1141-1144. doi:10.1021/ol0530272

29. Zhang, L.-B.; Hao, X.-Q.; Zhang, S.-K.; Liu, K.; Ren, B.; Gong, J.-F.; Niu, J.-L.; Song, M.-P. J. Org. Chem. 2014, 79, 10399-10409. doi:10.1021/jo502005j

30. Zhang, L.-B.; Hao, X.-Q.; Zhang, S.-K.; Liu, Z.-J.; Zheng, X.-X.; Gong, J.-F.; Niu, J.-L.; Song, M.-P. Angew. Chem., Int. Ed. 2015, 54, 272-275. doi:10.1002/anie.201409751

31. Guo, X.-K.; Zhang, L.-B.; Wei, D.; Niu, J.-L. Chem. Sci. 2015, 6, 7059-7071. doi:10.1039/C5SC01807B

32. Sauermann, N.; Meyer, T. H.; Tian, C.; Ackermann, L. J. Am. Chem. Soc. 2017, 139, 18452-18455. doi:10.1021/jacs.7b11025

33. Wang, G.-W.; Yuan, T.-T. J. Org. Chem. 2010, 75, 476-479. doi:10.1021/jo902139b

34. Jiang, T.-S.; Wang, G.-W. J. Org. Chem. 2012, 77, 9504-9509. doi:10.1021/jo301964m

35. Li, W.; Sun, P. J. Org. Chem. 2012, 77, 8362-8366. doi:10.1021/jo301384r

36. Kolle, S.; Batra, S. Org. Biomol. Chem. 2015, 13, 10376-10385. doi:10.1039/C5OB01500F

37. Zheng, Y.-W.; Ye, P.; Chen, B.; Meng, Q.-Y.; Feng, K.; Wang, W.; Wu, L.-Z.; Tung, C.-H. Org. Lett. 2017, 19, 2206-2209. doi:10.1021/acs.orglett.7b00463

38. Yin, Z.; Jiang, X.; Sun, P. J. Org. Chem. 2013, 78, 10002-10007. doi:10.1021/jo401623j

39. Shi, S.; Kuang, C. J. Org. Chem. 2014, 79, 6105-6112. doi:10.1021/j05008306

40. Li, S.; Zhu, W.; Gao, F.; Li, C.; Wang, J.; Liu, H. J. Org. Chem. 2017, 82, 126-134. doi:10.1021/acs.joc.6b02257

41. Yin, X.-S.; Li, Y.-C.; Yuan, J.; Gu, W.-J.; Shi, B.-F. Org. Chem. Front. 2015, 2, 119-123. doi:10.1039/C4QO00276H 
42. Lu, W.; Xu, H.; Shen, Z. Org. Biomol. Chem. 2017, 15, 1261-1267. doi:10.1039/C6OB02582J

43. Yoshikai, N. Bull. Chem. Soc. Jpn. 2014, 87, 843-857. doi:10.1246/bcsj.20140149

44. Fallon, B. J.; Derat, E.; Amatore, M.; Aubert, C.; Chemla, F.; Ferreira, F.; Perez-Luna, A.; Petit, M. J. Am. Chem. Soc. 2015, 137, 2448-2451. doi:10.1021/ja512728f

45. Moselage, M.; Li, J.; Ackermann, L. ACS Catal. 2016, 6, 498-525. doi:10.1021/acscatal.5b02344

46. Wei, D.; Zhu, X.; Niu, J.-L.; Song, M.-P. ChemCatChem 2016, 8, 1242-1263. doi:10.1002/cctc.201600040

47. Tan, G.; He, S.; Huang, X.; Liao, X.; Cheng, Y.; You, J. Angew. Chem., Int. Ed. 2016, 55, 10414-10418. doi:10.1002/anie. 201604580

48. Kalsi, D.; Barsu, N.; Sundararaju, B. Chem. - Eur. J. 2018, 24 , 2360-2364. doi:10.1002/chem.201705710

49. Du, C.; Li, P.-X.; Zhu, X.; Suo, J.-F.; Niu, J.-L.; Song, M.-P. Angew. Chem., Int. Ed. 2016, 55, 13571-13575. doi:10.1002/anie.201607719

50. Zhang, J.; Chen, H.; Lin, C.; Liu, Z.; Wang, C.; Zhang, Y. J. Am. Chem. Soc. 2015, 137, 12990-12996. doi:10.1021/jacs.5b07424

51. Wang, Y.; Du, C.; Wang, Y.; Guo, X.; Fang, L.; Song, M.-P.; Niu, J.-L.; Wei, D. Adv. Synth. Catal. 2018, 360, 2668-2677. doi:10.1002/adsc.201800036

\section{License and Terms}

This is an Open Access article under the terms of the Creative Commons Attribution License (http://creativecommons.org/licenses/by/4.0). Please note that the reuse, redistribution and reproduction in particular requires that the authors and source are credited.

The license is subject to the Beilstein Journal of Organic Chemistry terms and conditions:

(https://www.beilstein-journals.org/bjoc)

The definitive version of this article is the electronic one which can be found at:

doi:10.3762/bjoc. 14.183 